\title{
Menkes disease diagnosed by a novel ATP7A frameshift mutation in a patient with infantile spasms - a case report
}

\author{
Jinrong $\mathrm{Li}^{1,2}$, Ruolan $\mathrm{Hu}^{1,2}$, Jialing Wang ${ }^{1,2}$, Ruixin Yu ${ }^{1,2}$, Fei Xiong ${ }^{1,2}$, Mingyan Jiang ${ }^{1,2}$ \\ ${ }^{1}$ Department of Pediatrics, West China Second University Hospital of Sichuan University; ${ }^{2}$ Ministry of Education Key Laboratory of Birth Defects \\ and Related Diseases of Women and Children, Sichuan University, Chengdu, China \\ Correspondence to: Mingyan Jiang. No. 20, Section 3, Renmin South Road, Chengdu, China. Email: jiangmy0904@163.com.
}

\begin{abstract}
Menkes disease (MD) is a rare congenital copper deficiency disease caused by an adenosine triphosphatase copper transporting alpha $(A T P 7 A)$ gene mutation. It is a progressive and systemic disease that primarily involves the central nervous system and connective tissues. The clinical manifestation of these patients with MD is curly hair, progressive muscle tone reduction, and convulsions, and often leads to death in early infancy. Herein, we present a case of a 9-month-old Chinese male who displayed developmental regression, followed by convulsions, which were characterized by infantile spasms (ISs). The proband also had curly hair, hypopigmented skin, cutis laxa, decreased muscle tone, and micrognathia. The patient's ceruloplasmin levels were below the reference values. Brain magnetic resonance imaging (MRI) showed abnormal signals bilaterally that were symmetrically distributed in the caudate nucleus, globus pallidus, and subcortical white matter of the temporal parietal cortex, white matter in the anterior and posterior corners of the ventricles and the anterior limb of the internal capsule. The electroencephalograph (EEG) showed hypsarrhythmia. Genetic testing revealed a novel frameshift mutation in the ATP7A gene exon 13 and premature termination codon. Copper replacement therapy was initiated after the delayed diagnosis was established. However, the patient still died several months later due to disease progression. Our case reveals a novel frameshift mutation of the $A T P 7 A$ gene, which expands the gene spectrum of MD. The infants with uncontrollable convulsions, regressive development, curly hair, MD should be considered at early stage and also need the further genetic analysis to confirm MD finally. The correct and timely diagnosis and initiating copper replacement therapy may improve the prognosis.
\end{abstract}

Keywords: $A T P 7 A$; infantile spasm (IS); Menkes disease (MD); copper replacement therapy; case report

Submitted May 15, 2021. Accepted for publication Jul 08, 2021.

doi: $10.21037 /$ tp-21-275

View this article at: https://dx.doi.org/10.21037/tp-21-275

\section{Introduction}

Menkes disease [MD (OMIM 309400)] is an X-linked recessive genetic disease that involves multiple systems and is characterized by a disturbance in copper metabolism. $\mathrm{MD}$ is caused by the dysfunction of copper-transporting adenosine triphosphatase (ATPase) (1). ATP7A is the pathogenic gene that encodes for the ATPase $\mathrm{Cu}++$ transporting alpha polypeptide. A mutation in the $A T P 7 A$ gene can cause copper metabolism disorders, affect a variety of copper-dependent enzymes, and lead to progressive neurodegeneration and connective tissue abnormalities (2).
The incidence of MD is low (1/100,000-1/250,000) (3). At present, global reports of MD have generally involved sporadic cases. Due to the low incidence, insidious onset, and lack of knowledge, the MD is always missed and delayed diagnosed.

Convulsions is one of the main clinical characteristics of MD. Infantile spasms (ISs) is a typical type of convulsions in patients with MD (4) but always be ignored. Our patient is the first MD case with ISs in China with a novel frameshift mutation in the $A T P 7 A$ gene which expand the genetic spectrum and phenotype among Chinese patients.

We present the following article in accordance with 

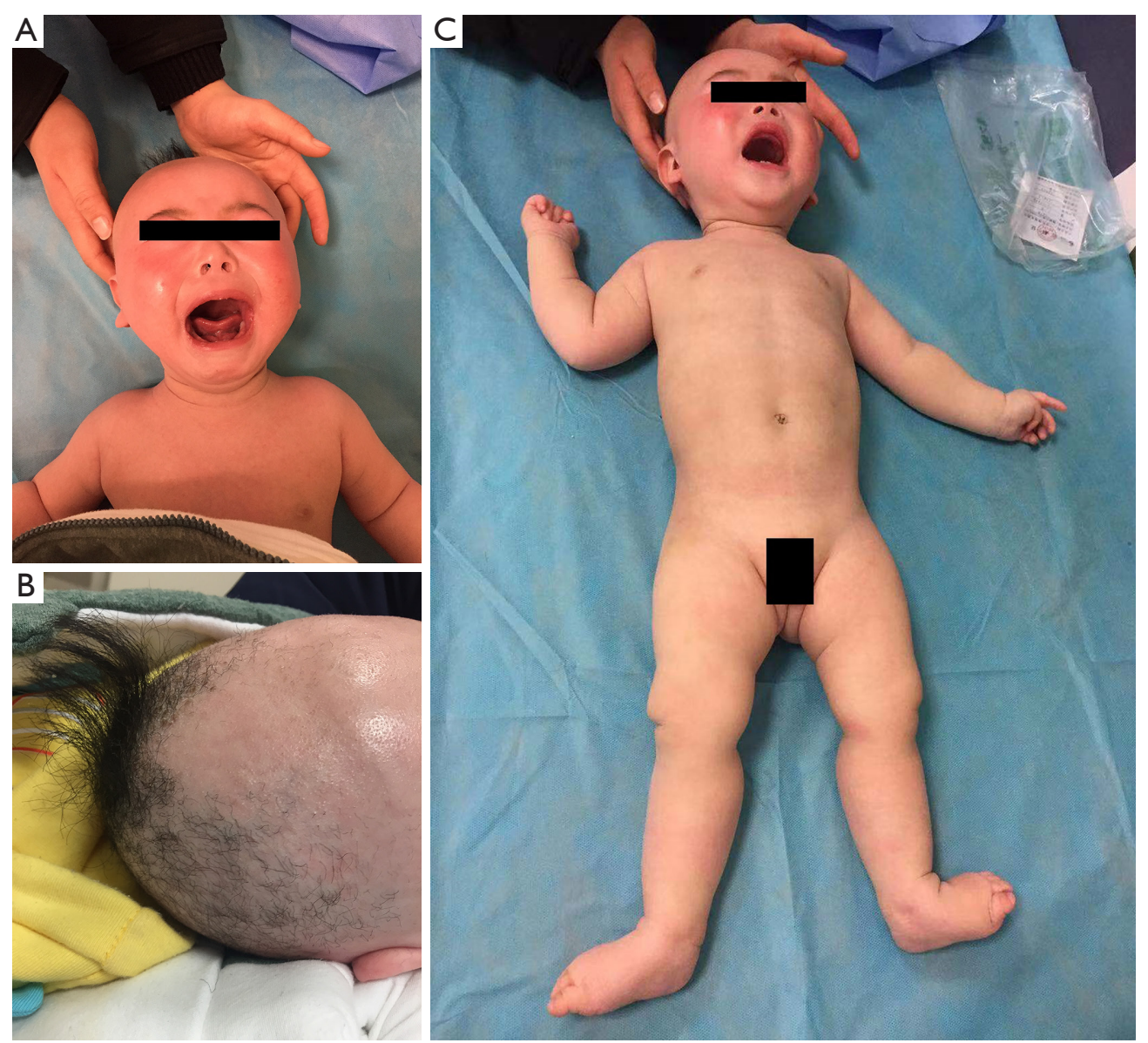

Figure 1 Photographs of the patient. (A) Facial appearance and alopecia. (B) Kinky and fragile hair. (C) Hypopigmented skin, joint laxity, and hypotonia.

the CARE reporting checklist (available at https://dx.doi. org/10.21037/tp-21-275).

\section{Case presentation}

The patient, a 9-month-old male, was admitted to our hospital for convulsions. He had been displaying regression in development for 4 months and convulsions for 1 week. The patient firstly showed developmental regression at 4 months ago (when aged 5-month-old), which manifested as an instability in raising his head and disability of gazing, laughing, or turning over. Convulsions occurred 1 week before admission (4-5 times daily), and most convulsions occurred before sleeping with regular nodding and hugging (pattern of ISs). The patient was diagnosed with West syndrome (also named as ISs) and Wilson's disease at other hospital and was treated with phenobarbital diazepam, and adrenocorticotropic hormone. However, the effect of the treatment was not satisfactory, and the convulsions was still out of control.

The patient was delivered spontaneously at 36 weeks' gestation with a birth weight of $3,000 \mathrm{~g}$, a length of $50 \mathrm{~cm}$, and Apgar scores of 9, 10, and 10 at 0,1 , and 5 minutes respectively. No abnormalities were noted during the perinatal period. The physical examination revealed hypopigmented skin, skin and joint laxity, hypotonia, kinky and fragile hair, tendon reflexes that could not be elicited, and bilateral positive Babinski signs (see Figure 1). Routine blood, biochemical, and cerebrospinal fluid testing were all negative. The patient had a ceruloplasmin level of $43 \mathrm{mg} / \mathrm{L}$ (normal range, 190-670 mg/L). An electroencephalograph (EEG) showed hypsarrhythmia. Brain magnetic resonance imaging (MRI) showed abnormal signals with a symmetric bilateral distribution in the caudate nucleus, globus pallidus, and subcortical white matter of the temporal parietal cortex, white matter in the anterior and posterior 


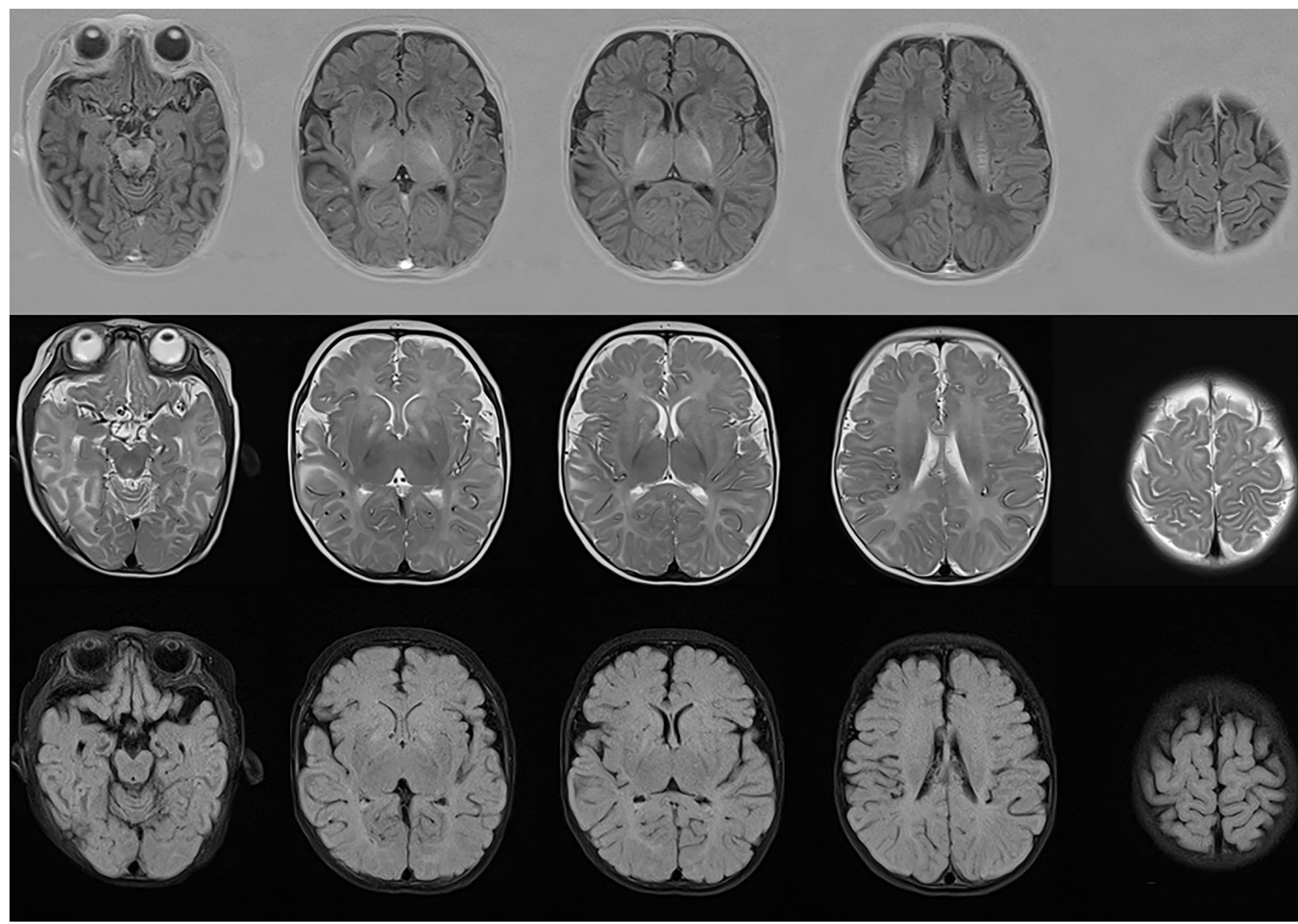

Figure 2 Brain MRI radiological findings: abnormal signals distributed bilaterally and symmetrically in the caudate nucleus, globus pallidus, and subcortical white matter of the temporal parietal cortex, white matter in the anterior and posterior corners of the ventricles and the anterior limb of the internal capsule. MRI, magnetic resonance imaging.

corners of the ventricles and anterior limb of the internal capsule (see Figure 2). A peripheral nerve examination and somatosensory to evoke potential were normal. During his early life (before 5 months old), the patient showed normal growth and development. He was able to raise his head 90 degrees for $2 \mathrm{~min}$ and follow sounds and gazes to a normal degree as evaluated by the Neonatal Behavioral/ Neurological Assessment (NA/NB) and the Bayley Scales of Infant Development. However, after 5 months of age, the patient began to show instability of raising head steadily. And at 6 months of age, he could not gaze, laugh, turn over or even raise up head. The results of a Peabody examination at 9 months of age were as follows: gross motor quotient 45 , fine motor quotient 52 , and total motor quotient 44 . All procedures performed in studies involving human participants were conducted in accordance with the ethical standards of the institutional and/or national research committee(s) and the Helsinki Declaration (as revised in 2013). Written informed consent was obtained from the patient's parents.

\section{Molecular findings}

The gene sequencing (see Figure 3) indicated a hemizygous duplication mutation in exon 13 in Xq21 (c.2780dupA) which led to a translation frameshift. And this frameshift of the gene caused a disorder of the encoded protein from the 928th amino acid residue [Ala (p.Ala928fs)] and also led to a premature termination codon, thus affecting the activity of P-ATPase. The mutation was confirmed to be a novel pathogenic mutation after comparison with the gene pools (http://www.hgmd.cf.ac.uk/ac/gene/php?gene=ATP7A and http://www.LOVD.nl/ATP7A). The $A T P 7 A$ genes of the parents were normal. 


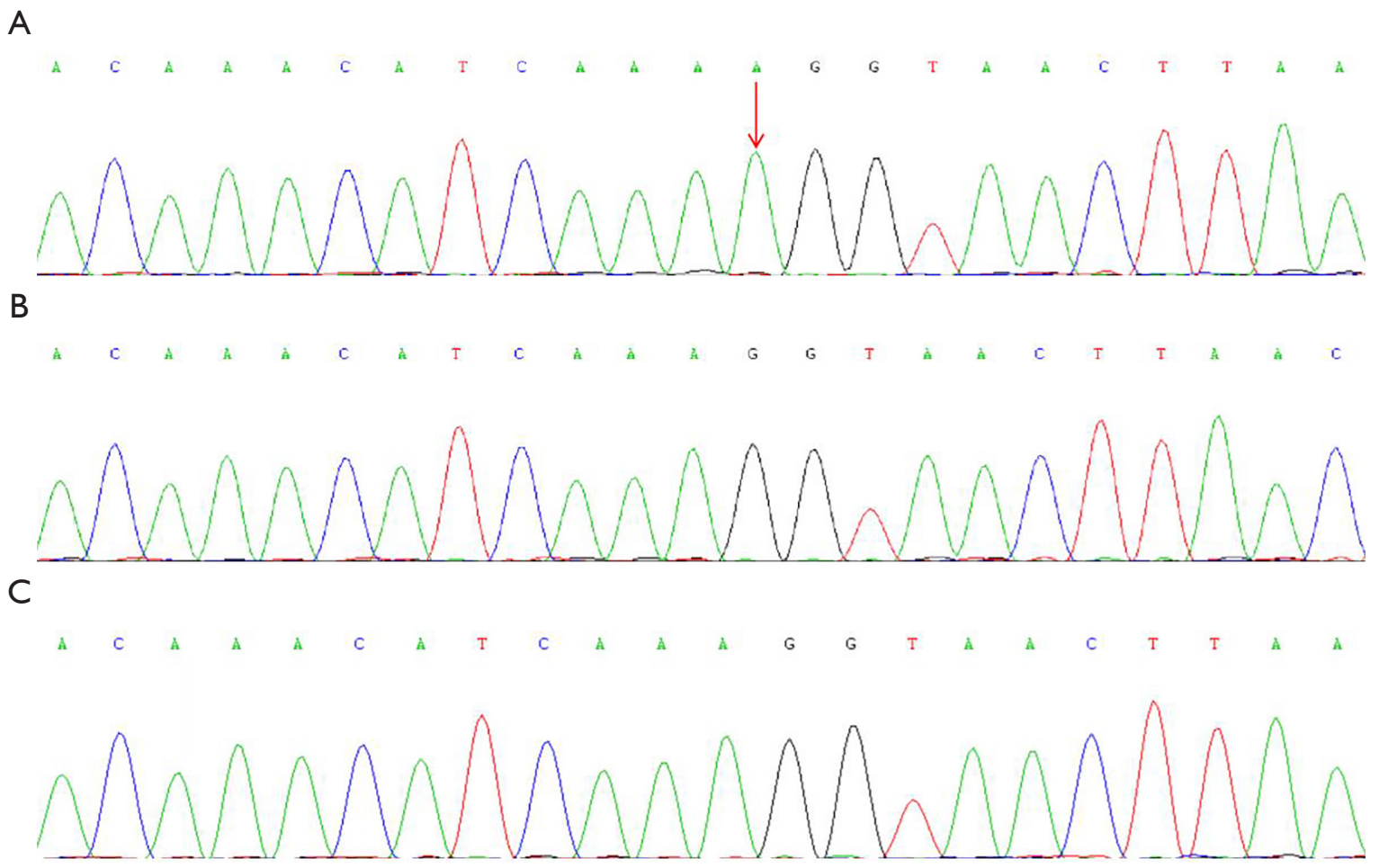

Figure 3 Sequencing results of the $A T P 7 A$ (adenosine triphosphatase copper transporting alpha) mutation. (A) A gene analysis revealed a c.2780dupA at exon 13 of the patient (p.Ala928fs). (B) $A T P 7 A$ gene sequencing of the patient's father was normal. (C) $A T P 7 A$ gene sequencing of the patient's mother was normal.

\section{Diagnosis and treatment}

Based on the typical clinical manifestations of the patient (i.e., repeated convulsions, curly hair, hypopigmented skin, skin and joint laxity, and hypotonia), the novel frameshift mutation in the $A T P 7 A$ gene, the brain MRI and EEG findings, the patient was diagnosed with classic MD. Therefore, the copper replacement therapy and a variety of anti-epileptic drugs (oxcarbazepine and levetiracetam) were initiated.

\section{Follow-up}

The patient showed improved convulsion control after the use of copper histidine from Japan and anti-epileptic drugs (the frequency of conculsions decreased to 2-4 times for 2 weeks), but rehabilitation did not improve the patient's development regression. The patient was dead 11 months later due to a 25-minute status convulsion and sepsis.

\section{Discussion}

The main clinical symptoms of MD are progressive neurodegeneration, abnormal connective tissues, and special curly hair. MD can be classified as classic, mild, or occipital angle syndrome $(2,5)$. And we can make a rapid judgement of the prognosis by different types of MD. Among these syndromes, classic MD often occurs during the neonatal period. The onset of classic MD manifests as hypoglycemia, hypothermia, persistent hyperbilirubinemia, and feeding difficulties, after which the nervous system gradually deteriorates, resulting in developmental regression, developmental retardation, convulsions and always died within 3 years old. Otherwise, the patient with mild MD or occipital angle syndrome can survive to adulthood or even longer (5). The patient in this case exhibited the clinical features of classic MD with ISs as the prominent manifestation. An EEG showed hypsarrhythmia, and multiple anti-epileptic drugs were needed to control the convulsions. Convulsions constitute one of the main clinical features of classic MD, which may have different manifestations at different periods. In the early (approximately 3 months of age) and middle (3-8 months after onset) periods, a patient may experience 
uncontrollable focal seizures or ISs $(6,7)$. As the disease progresses, MD can gradually manifest as status epilepticus, tonic-clonic seizures, generalized tonic and clonic seizures, or other types of convulsions (4). The cause of convulsions in MD patients may be copper-mediated N-methyl-Daspartic acid receptor dysfunction, which enhances neuronal excitability and excitatory neurotoxic damage, thus leading to convulsions (6).

MD is a rare $\mathrm{X}$-linked recessive genetic disease. The pathogenic mechanism is abnormal copper metabolism caused by the mutation in the $A T P 7 A$ gene. The $A T P 7 A$ gene is located at $\mathrm{Xq} 13.3$, which has 23 exons, encodes 1,500 amino acids, and is widely expressed in the brain, kidneys, lungs, and muscles (1). The ATP7A gene encodes the energy-dependent $\mathrm{Cu}++$ transporting alpha polypeptide, which is a member of the $\mathrm{P}$-type coppertransporting ATPase family that constitutes a $\mathrm{Cu}++$ transporting channel, participates in $\mathrm{Cu}++$ binding and ATP hydrolyzation, and affects dopamine b hydroxylase and cytochrome c oxidase activity by affecting $\mathrm{Cu}++$ metabolism (8), thereby changing the cellular response to oxidative stress, lysosomal transporting, signal transduction, mitochondrial function, and immune responses, eventually leading to abnormal connective tissue, tortuosity of blood vessels, and fluffy hair (6). It has been reported that c.2179 g $>\mathrm{A}$ in Chinese patients may be the mutation hotspot of MD while deletions, insertions, or duplications in MD gene mutations also account for $33 \%$ of cases $(9,10)$. With the progress of molecular genetics technology and further research of $A T P 7 A$ gene, gene therapy will be the effective treatment in the future. Donsante et al. firstly confirmed that the long-term survival rate of high-dose gene therapy combined with intramuscular injection of copper histidine was up to $53.3 \%$ in MD mice, while the control group was $0 \%$. However, the efficacy and safety of gene therapy still need to be further confirmed by the clinical trials (11).

Whole exon sequencing revealed a novel mutation (c.2780dupA) in the ATP7A gene of the patient in this report, which led to the disorder of the 928 amino acid residue Ala (p.Ala928fs) and premature termination codon, thereby changing the ATPase activity. When compared to the Human Gene Mutation Database (www.hgmd.org) and the $A T P 7 A$ database in the Leiden Open Variation Database 3.0 (www.LOVD.nl/ATP7A), it was confirmed that the frameshift mutation in the current patient was an unreported novel pathogenic mutation; however, the onset of MD was relatively late and developmental regression occurred 5 months after birth. Thus, the patient's MD differed from that of classic MD which commonly occurs in the neonatal period. The pathogenesis of delayed classic MD of our patient may be due to unique genetic variation in this case. The severity of MD depends on the level of residual ATP7A function (12). At present, more than 300 different $A T P 7 A$ gene mutations have been identified (13), among which truncating variants cause severe classic MD that can lead to the early death of children (14). It has been reported that novel mutations result in a translation shift and premature termination codon, which may lead to the severe classic MD clinical phenotype. However, the actual clinical phenotype of the current patient was milder than that of the predicted genotype. Presumably, translation reinitiation may have occurred. Translation re-initiation can occur downstream of the premature termination codon and produce a partially functional ATP7A protein $(15,16)$. In this case, the onset of MD was late but the symptoms were typical, and might be similar with the researches from Wada T and Paulsen M $(15,16)$, which is related to the residual function of ATP7A. However, whether hemizygous duplication mutation of our proband affects messenger ribonucleic acid splicing and the level of ATP7A expression or not is unknown. Functional analyses need to be conducted in the future to further explain the effects of mutations on the clinical phenotype.

The patient was misdiagnosed with ISs and Wilson's disease at early infancy. MD and Wilson's disease are both inherited disorders of copper metabolism, which can cause varying degrees of nervous system degeneration and convulsions $(2,6)$. However, the patient had typical clinical phenotype including unique curly hair, a significantly reduced ceruloplasmin level and ISs, but with absence of Fleischer-Kayser ring and were consistent with the symptoms of MD patients reported in the literatures and distinguished from ISs and Wilson's disease, which finally diagnosed as MD by ATP7A gene mutation $(17,18)$. The patient with hair changes, connective tissue abnormalities, progressive central nervous system degeneration should be considered as clinically suspected cases. Biochemical screening has great advantages in rapid diagnosis, and molecular technology make a definite diagnosis later. Low level of serum copper and ceruloplasmin highly indicates $\mathrm{MD}$ and the biochemical screening of copper metabolism can be the early screening method. There are three major treatments for MD including copper supplement, antiepileptic treatment and supportive treatment $(6,19)$ with limited effect.

Convulsions in majority of patients with $\mathrm{MD}$ are difficult 
to control and show resistance to anti-epileptic drugs $(6,7)$. At present, copper histidine is widely used as an alternative treatment; however, the value of the supplementation of copper is limited unless the treatment is started within a few days of birth $(6,19)$. The delayed use of copper histidine is not effective in improving neurologic degeneration and convulsions (4). Early copper replacement therapy is particularly suitable for patients with ATP7A protein residual transporting function, which can help improve survival and reduce neurological damage $(6,19)$. Thus, early diagnosis and treatment are particularly important for MD patients.

In conclusion, MD is a rare disease with developmental regression, intractable epilepsy, and curly hair. For infants with these phenotypes, the suspicion of MD should be conducted and rapid biochemical test of serum copper and ceruloplasmin need to be done as an early screening method. But these clinical symptoms of MD also overlapped with some genetic diseases especially other copper metabolic diseases can hardly be the guideline for $M D$, so the further gene analysis also should be done in order to make the final diagnosis of MD. In the present study, a novel $A T P 7 A$ mutation associated with $M D$ was revealed, which expands the $A T P 7 A$ gene spectrum. In order to improve the outcomes of these patient and prolong the survival age, the early diagnosis and timely copper replacement therapy are crucial.

\section{Acknowledgments}

The authors would like to thank the family members of the patient for providing blood samples, co-operating with the therapeutic strategies, and agreeing to participate in this study. Funding: Jinrong Li received funding from the Youth Innovation Project of Medical Research in Sichuan Province (Q18015).

\section{Footnote}

Reporting Checklist: The authors have completed the CARE reporting checklist. Available at https://dx.doi. org/10.21037/tp-21-275

Conflicts of Interest: All authors have completed the ICMJE uniform disclosure form (available at https://dx.doi. org/10.21037/tp-21-275). The authors have no conflicts of interest to declare.

Ethical Statement: The authors are accountable for all aspects of the work in ensuring that questions related to the accuracy or integrity of any part of the work are appropriately investigated and resolved. All procedures performed in studies involving human participants were conducted in accordance with the ethical standards of the institutional and/or national research committee(s) and the Helsinki Declaration (as revised in 2013). Written informed consent was obtained from the patient's parents.

Open Access Statement: This is an Open Access article distributed in accordance with the Creative Commons Attribution-NonCommercial-NoDerivs 4.0 International License (CC BY-NC-ND 4.0), which permits the noncommercial replication and distribution of the article with the strict proviso that no changes or edits are made and the original work is properly cited (including links to both the formal publication through the relevant DOI and the license). See: https://creativecommons.org/licenses/by-nc-nd/4.0/.

\section{References}

1. Vulpe C, Levinson B, Whitney S, et al. Isolation of a candidate gene for Menkes disease and evidence that it encodes a copper-transporting ATPase. Nat Genet 1993;3:7-13.

2. Caicedo-Herrera G, Candelo E, Pinilla J, et al. Novel ATP7A gene mutation in a patient with Menkes disease. Appl Clin Genet 2018;11:151-5.

3. Tønnesen T, Kleijer WJ, Horn N. Incidence of Menkes disease. Hum Genet 1991;86:408-10.

4. Verrotti A, Cusmai R, Darra F, et al. Epilepsy in Menkes disease: an electroclinical long-term study of 28 patients. Epilepsy Res 2014;108:1597-603.

5. Tümer Z, Møller LB. Menkes disease. Eur J Hum Genet 2010;18:511-8.

6. Prasad AN, Levin S, Rupar CA, et al. Menkes disease and infantile epilepsy. Brain Dev 2011;33:866-76.

7. Bahi-Buisson N, Kaminska A, Nabbout R, et al. Epilepsy in Menkes disease: analysis of clinical stages. Epilepsia 2006;47:380-6.

8. Mak BS, Chi CS, Tsai CR. Menkes gene study in the Chinese population. J Child Neurol 2002;17:250-2.

9. Cao B, Yang X, Chen Y, et al. Identification of novel ATP7A mutations and prenatal diagnosis in Chinese patients with Menkes disease. Metab Brain Dis 2017;32:1123-31.

10. Møller LB, Bukrinsky JT, Mølgaard A, et al. Identification and analysis of 21 novel disease-causing amino acid substitutions in the conserved part of ATP7A. Hum Mutat 
2005;26:84-93.

11. Donsante A, Yi L, Zerfas PM, et al. ATP7A gene addition to the choroid plexus results in long-term rescue of the lethal copper transport defect in a Menkes disease mouse model. Mol Ther 2011;19:2114-23.

12. Ojha R, Prasad AN. Menkes disease: what a multidisciplinary approach can do. J Multidiscip Healthc 2016;9:371-85.

13. Stenson PD, Mort M, Ball EV, et al. The Human Gene Mutation Database: 2008 update. Genome Med 2009;1:13.

14. Tümer $Z$. An overview and update of ATP7A mutations leading to Menkes disease and occipital horn syndrome. Hum Mutat 2013;34:417-29.

15. Wada T, Haddad MR, Yi L, et al. A novel two-nucleotide deletion in the ATP7A gene associated with delayed

Cite this article as: Li J, Hu R, Wang J, Yu R, Xiong F, Jiang M. Menkes disease diagnosed by a novel ATP7A frameshift mutation in a patient with infantile spasms-a case report. Transl Pediatr 2021;10(7):1965-1971. doi: 10.21037/ tp-21-275 infantile onset of Menkes disease. Pediatr Neurol 2014;50:417-20.

16. Paulsen M, Lund C, Akram Z, et al. Evidence that translation reinitiation leads to a partially functional Menkes protein containing two copper-binding sites. Am J Hum Genet 2006;79:214-29.

17. Manara R, D'Agata L, Rocco MC, et al. Neuroimaging Changes in Menkes Disease, Part 1. AJNR Am J Neuroradiol 2017;38:1850-7.

18. Bindu PS, Taly AB, Kothari S, et al. Electro-clinical features and magnetic resonance imaging correlates in Menkes disease. Brain Dev 2013;35:398-405.

19. Vairo FPE, Chwal BC, Perini S, et al. A systematic review and evidence-based guideline for diagnosis and treatment of Menkes disease. Mol Genet Metab 2019;126:6-13. 